\title{
Cross Talk Between Ferroptosis and Cerebral Ischemia
}

\author{
Xu She, Bin Lan, Haomei Tian* and Biao Tang* \\ Department of Physiology, Hunan University of Chinese Medicine, Changsha, China
}

Recently, ferroptosis has been revealed as a new form of regulated cell death. Distinct from apoptosis and necrosis, ferroptosis is evoked by iron-dependent lipid peroxidation. Furthermore, the metabolism of iron, lipids, and amino acids plays a significant regulatory role in ferroptosis, which can be reversed by glutathione peroxidase 4 and ferroptosis suppressor protein 1. Ferroptosis is implicated in the onset and development of numerous neurological diseases. Emerging studies have reported that ferroptosis induces and aggravates brain tissue damage following cerebral ischemia,

OPEN ACCESS

Edited by:

Anwen Shao,

Second Affiliated Hospital, School of Medicine, Zhejiang University,

China

Reviewed by:

Peiying Li,

Renji Hospital, Shanghai Jiao Tong University School of Medicine, China

Jianmin Zhang,

Zhejiang University, China,

Danfeng Lin,

Zhejiang University, China, in collaboration with reviewer $J Z$

*Correspondence:

Biao Tang

biaotang@hnucm.edu.cn

Haomei Tian

451358104@qq.com

Specialty section:

This article was submitted to

Neurodegeneration,

a section of the journal

Frontiers in Neuroscience

Received: 20 April 2020

Accepted: 01 July 2020

Published: 06 August 2020

Citation:

She $X$, Lan B, Tian H and Tang B

(2020) Cross Talk Between

Ferroptosis and Cerebral Ischemia.

Front. Neurosci. 14:776.

doi: 10.3389/fnins.2020.00776 whereas inhibition of ferroptosis dramatically attenuates induced damage. In this review, we have summarized the mechanistic relationship between ferroptosis and cerebral ischemia, including through iron overload, downregulation of glutathione peroxidase 4, and upregulation of lipid peroxidation. Although considerable attention has been paid to the effect of ferroptosis on cerebral ischemic injury, specific mechanisms need to be experimentally confirmed, including how cerebral ischemia induces ferroptosis and how ferroptosis deteriorates cerebral ischemia.

\section{Keywords: ferroptosis, cerebral ischemia, iron metabolism, amino acid metabolism, lipid metabolism}

\section{INTRODUCTION}

Ferroptosis is a type of regulated cell death dependent on iron. In 2012, ferroptosis was first used to describe cell death induced by erastin, a small molecule that inhibits cystine uptake and leads to reduced glutathione (GSH) production, as well as the inactivation of glutathione peroxidase 4 (GPX4) (Dixon et al., 2012). Ferroptosis relies on intracellular iron rather than other metallic elements and could be inhibited by iron chelators and antioxidants, such as vitamin E. Moreover, it is distinct from other forms of cell death, including apoptosis, necroptosis, and autophagydependent cell death, in terms of morphology, biochemistry, and genetics (Dixon et al., 2012). Furthermore, inhibitors of apoptosis, necroptosis, or autophagy are incapable of suppressing ferroptosis (Dolma et al., 2003).

Abbreviations: 12/15-LOX, 12/15-lipoxygenase; AA, arachidonic acid; ACSL4, acyl-CoA synthetase long-chain family member 4; AdA, adrenic acid; CEF, ceftriaxone-; $\mathrm{CoQ}_{10}$, coenzyme $\mathrm{Q}_{10}$; $\mathrm{CoQ}_{10} \mathrm{H}_{2}$, reduced coenzyme $\mathrm{Q}_{10}$; DAMP, damage-associated molecular pattern; DMT1, divalent metal transporter 1; FPN, ferroportin1; FSP1, ferroptosis suppressor protein 1; FT, ferritin; GPX4, glutathione peroxidase 4; GSH, reduced glutathione; GSSG, oxidized glutathione; HIF-1 $\alpha$, hypoxia-inducible factor-1 $\alpha$; HTF, holotransferrin; IS, ischemic stroke; LIP, labile iron pool; L-O, alkyl oxygen free radicals; L-OH, phospholipids-H; L-OOH, phospholipids-OH; LOX, lipoxygenase; LPCAT3, lysophosphatidylcholine acyltransferase 3; miR, miRNA; MVA, mevalonate; NAC, N-acetylcysteine; NADPH, nicotinamide adenine dinucleotide phosphate; NMDAR, N-methyl-D-aspartate receptor; Nrf2, nuclear factor erythroid-2-related factor 2; PE, phosphatidylethanolamine; PUFAs, polyunsaturated fatty acids; ROS, reactive oxygen species; system xc-, cystine/glutamate reverse transporters; SIH, salicylaldehyde isonicotinoyl hydrazine; STEAP3, six-transmembrane epithelial antigen of the prostate 3; TF, transferrin; TFR1, transferrin receptor 1 . 
The main characteristic of ferroptosis is death due to the accumulation of lethal levels of iron-dependent lipid peroxides (for review, see Michael and Brent, 2017). Previous studies have shown that ferroptosis has pathological roles in a wide variety of diseases, including neurodegenerative diseases, cancer, ischemiareperfusion injury, stroke, and traumatic brain injury (for review, see Conrad et al., 2016; Newton et al., 2016; Li et al., 2017; Toyokuni et al., 2017; Zille et al., 2017).

\section{DISTINCT MORPHOLOGICAL FEATURES OF FERROPTOSIS AS COMPARED TO APOPTOSIS, NECROSIS, AND AUTOPHAGY}

Morphologically, ferroptotic cells exhibit typical features of necrosis, with no apoptosis features. Importantly, ferroptosis is accompanied by a series of morphological changes in the mitochondria, including cristae reduction, membrane coagulation, and rupture of the outer membrane, whereas in other forms of cell death, the mitochondria are usually swollen (Dixon et al., 2012). Moreover, in cancer cells treated with ferroptosis inducers, like erastin, the structural integrity of the nucleus is maintained, with no nucleation or chromatin edge observed. These differences in morphological features are important to distinguish ferroptosis from apoptosis, necrosis, and autophagy (Xie et al., 2016).

Biochemical processes related to ferroptosis include metabolisms involving iron, amino acids, or polyunsaturated fatty acids (PUFAs), as well as the biosynthesis of GSH, phospholipids, and nicotinamide adenine dinucleotide phosphate, and coenzyme $\mathrm{Q}_{10}$ (for review, see Stockwell et al., 2017).

\section{IRON, AMINO ACID, AND LIPID METABOLISMS ARE DEEPLY INVOLVED IN THE MOLECULAR MECHANISMS OF FERROPTOSIS}

Notably, mechanisms associated with ferroptosis are complex and mainly include three factors: iron, amino acid, and lipid metabolisms. Iron is an essential element in ferroptosis. A robust increase in iron levels can trigger the accumulation of lipid peroxides and reactive oxygen species (ROS), resulting in ferroptosis (Yan and Zhang, 2019).

Amino acid metabolism is closely associated with ferroptosis. In most cases, the intake of cysteine occurs via cystine/glutamate reverse transporters (system $\mathrm{xc}$-), which function in the intracellular environment to synthesize GSH and maintain the antioxidant activity of GPX4.

Lipoxygenase transforms esterified PUFAs located in the cell membrane into lipid peroxides (Yang et al., 2016). Reportedly, lipoxygenase is a crucial agent playing a direct role in ferroptosis (Yang et al., 2016). Coenzyme $Q_{10}$, a downstream effector of $\beta$-hydroxy $\beta$-methylglutaryl-CoA
(HMG-CoA) reductase, which synthesizes mevalonic acid (MVA), is an endogenous ferroptosis inhibitor possessing profound antioxidant effects in the intracellular environment (Shimada et al., 2016). Suppression of this coenzyme can potentiate the accumulation of lipid peroxides, resulting in cell death (Shimada et al., 2016). According to the latest research, FSP1 represses lipid peroxidation by reducing coenzyme $\mathrm{Q}_{10}$ through $\mathrm{NAD}(\mathrm{P}) \mathrm{H}$, thus eliciting an inverse role in the occurrence of ferroptosis (Bersuker et al., 2019; Doll et al., 2019). These results indicate that in ferroptosis, cell death is characterized by lipid peroxidation. Conceivably, the interventions of ferroptosis by targeting lipid peroxidation are reasonable.

\section{Iron Metabolism}

Iron overload is a key event in ferroptosis, with $\mathrm{Fe}^{2+}$ generating lipid ROS through the Fenton reaction (for review, see Toyokuni et al., 2017). Furthermore, $\mathrm{Fe}^{2+}$ constitutes the lipoxygenase (LOX) catalytic subunit that initiates lipid peroxidation. Both these functions of $\mathrm{Fe}^{2+}$ are crucial for the onset of ferroptosis. There are two transport mechanisms for the entry of non-heme iron into cells: transferrin (TF)-dependent and TF-independent mechanisms. Several mechanisms occur independently of TF; for example, extracellular $\mathrm{Fe}^{2+}$ enters cells through membrane divalent metal transporter 1 (DMT1). However, most plasma $\mathrm{Fe}^{3+}$ exists in the complete form with $\mathrm{TF}$ and is transformed into holotransferrin (for review, see Hentze et al., 2010). After endocytosis, holotransferrin binds to TF receptor 1 (TFR1) on the cell membrane surface and subsequently moves to endosomes. In the acidic environment of the endosome, $\mathrm{Fe}^{3+}$ is released from TF and converted into $\mathrm{Fe}^{2+}$ through oxidation-reduction. Consequently, $\mathrm{Fe}^{2+}$ is transported to the labile iron pool in the cytoplasm through DMT1 on the endosome membrane, where it forms a complex that functions as a physiological or pathological element (for review, see Richardson and Ponka, 1997; Hentze et al., 2010). The secretion or storage of excessive $\mathrm{Fe}^{2+}$ in the ferritin complex blocks the formation of hydroxyl radicals from $\mathrm{H}_{2} \mathrm{O}_{2}$, thus preventing ROS production (for review, see Richardson and Ponka, 1997). Some $\mathrm{Fe}^{2+}$ ions are transported outside the cell membrane through ferroportin1 (FPN1) residing in the cell membrane; therefore, the intracellular iron concentration is considered appropriate under physiological conditions (Donovan et al., 2000).

\section{Amino Acid Metabolism}

A study has shown that the entrance of cystine into the cytoplasm could be the mainstay of ferroptotic suppression, through the maintenance of intracellular GSH levels (Le et al., 2015). In contrast, the decrease in GSH synthesis contributes to ferroptosis (Philpott and Ryu, 2014). The translocation of extracellular cystine and intracellular glutamate by system $\mathrm{xc}$ - is driven by the high glutamate concentration in the intracellular environment, rather than ATP, at a ratio of $1: 1$, and the repression of system xc- directly results in ferroptosis (Dixon et al., 2012). Following the injury of brain 
tissue, system xc- is impacted by the high concentration of extracellular glutamate (Jose et al., 2014; for review, see Bridges et al., 2012). After crossing the cytomembrane, cystine is degraded into cysteine and, subsequently, cysteine is converted into $\gamma$-glutamyl-cysteine by glutamyl-cysteine ligase. Then, $\gamma$ glutamyl-cysteine combines with glycine to form GSH, which in turn is activated by glutathione synthase (for review, see Aoyama and Nakaki, 2015).

In recent years, the suppression of GPX4, a key anti-lipid peroxidation enzyme, has been considered a committed step of ferroptosis, either through a direct (such as via covalent inhibition of RSL3 and its related molecules; Yang et al., 2014) or indirect (such as via GSH depletion) manner, provoking the unacceptable upregulation of phospholipid-OH and alkyl oxygen free radicals, causing severe membrane damage and ferroptosis (Dixon et al., 2012; Yan and Zhang, 2019). GPX4 activates phospholipid$\mathrm{OH}$ to phospholipid-H via a reduction reaction, with the consumption of two molecules of GSH (Yan and Zhang, 2019). Additionally, GSH is transformed into oxidized glutathione (Yan and Zhang, 2019). Oxidized glutathione is then reduced to GSH by the nicotinamide adenine dinucleotide phosphate-dependent glutathione reductase, entering the next cycle of reduction reaction (Leslie and Scott, 2018). The oxidized phospholipid-OH is converted into massive alkyl oxygen free radicals with highly reactivity initiated by intracellular free $\mathrm{Fe}^{2+}$ (for review, see Gaschler and Stockwell, 2016). The latter induces adverse effects on adjacent PUFAs through a cascade of chain reactions. Eventually, an extensive spectrum of membrane damage occurs, decreasing cell viability and even causing death (for view see Philpott and Ryu, 2014).

Furthermore, as a binding ligand for free $\mathrm{Fe}^{2+}$ in the labile iron pool, GSH can prevent $\mathrm{Fe}^{2+}$ from reacting with $\mathrm{H}_{2} \mathrm{O}_{2}$. Conversely, highly cytotoxic hydroxyl radicals are generated by the lack of GSH (for review see Philpott and Ryu, 2014).

\section{Lipid Metabolism}

Lipid metabolism is closely related to ferroptosis (for review, see Stockwell et al., 2017). Considering that PUFAs are sensitive to oxidants, they function as primary substrates of lipid peroxidation related to ferroptosis, especially arachidonic acid (AA) and adrenic acid (AdA) (Yan and Zhang, 2019). First, PUFAs are esterified with membrane phospholipids, mainly phosphatidylethanolamine (PE). The esterification reaction is catalyzed by acyl-CoA synthetase long-chain family member 4 (ACSL4) and lysophosphatidylcholine acyltransferase 3 (LPCAT3), resulting in the formation of AA/AdA-PE. Next, LOX converts PE-AA/AdA into $\mathrm{PE}-\mathrm{AA} / \mathrm{AdA}-\mathrm{OH}$ through oxidation, thereby inducing ferroptosis (Dixon et al., 2015; Yang et al., 2016; Doll et al., 2017; Kagan et al., 2017; Shintoku et al., 2017). Furthermore, the degree of ferroptosis can be determined by measuring the amount of peroxidized PUFAs and examining their subcellular localization (Stockwell et al., 2017). However, the specific mechanism through which lipid peroxidation mediates ferroptosis remains unknown (Eran et al., 2018).

\section{FERROPTOSIS EXERTS PROFOUND REGULATORY EFFECTS IN CEREBRAL ISCHEMIC BRAIN INJURY}

The relationship between ferroptosis, as a fundamental form of cell death in the nervous system, and neurological diseases has been reported in several diseases, including Alzheimer's disease, Parkinson's disease, Huntington's disease, amyotrophic lateral sclerosis, Friedreich's ataxia, traumatic brain injury, and periventricular leukomalacia (Chen et al., 2015; Do Van et al., 2016; Gascón et al., 2016; for review, see Skouta et al., 2014; Guiney et al., 2017; Hambright et al., 2017; Li et al., 2017; Zille et al., 2017).

Ischemic stroke (IS) is a major cause of death and disability worldwide. It is characterized by the sudden obstruction of local blood flow to the brain and results in irreversible damage and deficiency of nerve function (for review, see Lee et al., 1999). There are multiple mechanisms involved in ischemic neuronal injury, among which oxidative stress is important in the progression of ischemic injury. In ischemic brain tissue, the defense ability of the antioxidant system is weakened owing to ROS elevation, followed by the excessive production of oxygen free radicals, deactivation of antioxidant enzymes, and antioxidant depletion. This results in multiple deleterious events, including peroxidation of lipids and proteins, damage to nuclear DNA, and cell death (for review, see Chan, 2001). Moreover, it has been shown that oxidative damage in the ischemic brain tissue is continuous and even spreads to surrounding healthy tissues; however, treatment with antioxidants can noticeably attenuate cerebral ischemic damage (Imai et al., 2003). A recent study has reported that oxidative stress injury can induce ferroptosis and leads to neuronal death in neurodegenerative diseases (Chuanhong et al., 2018) and that BID plays an important role in the ferroptosis mechanism-induced oxidative stress (Neitemeier et al., 2017) and is hence possibly linked with cerebral ischemic damage. However, the role of ferroptosis remains unclear in cerebral ischemia and further research is needed. Multiple studies have revealed that ferroptosis plays a regulatory role in ischemic brain damage, mainly in neurons. However, the suppressive intervention of ferroptosis can exert neuroprotective effects (DeGregorio-Rocasolano et al., 2019). These findings imply that ferroptosis possesses the potential to serve as a therapeutic target for cerebral ischemia (Cheah et al., 2006; Gubern et al., 2013; Tuo et al., 2017; Yigitkanli et al., 2017).

Reportedly, ferroptosis exerts profound regulatory effects during ischemia-reperfusion brain injury. Additionally, ferroptosis has been detected in mouse models of cerebral ischemia. Administration of the ferroptosis inhibitors, liproxstatin-1 or ferrostatin-1 (Fer-1), after reperfusion, generated a markedly smaller infarct, thus alleviating cerebral injury (Tuo et al., 2017). Furthermore, studies have revealed that inhibition of ferroptosis can reduce ischemic brain damage in rats, suggesting that ferroptosis mediates the onset and 
development of IS and exacerbates cerebral injury (Alim et al., 2019; Guan et al., 2019; Lan et al., 2020).

Moreover, the inflammatory mechanism is probably responsible for the aggravation of cerebral ischemic injury mediated by ferroptosis. Typically, ferroptosis exerts a strong pro-inflammatory effect through the release of pro-inflammatory mediators, and this inflammatory response is the key mechanism of cerebral ischemic injury (for review, see Linkermann et al., 2014b). Although research in this field is limited, a recent review indicated that ferroptosis plays an important role in inflammation (Sun et al., 2020) and, reportedly, inflammation is activated in ferroptotic tissues in mouse kidney injury models (Linkermann et al., 2014a). Additionally, the relationship between inflammation and dead neurons was demonstrated in the GPX4-depleted model (Hambright et al., 2017). Both the abovementioned findings provided an experimental basis for the hypothesis.

\section{POTENTIAL REGULATORY MECHANISMS OF FERROPTOSIS IN CEREBRAL ISCHEMIA}

The association between ferroptosis and cerebral ischemia is complex. Several previous studies elucidated that the activation of ferroptosis following an ischemic episode involves iron overload (Tuo et al., 2017) and excitatory toxicity induced by the upregulation of $\mathrm{xCT}$ (one heterodimer composed of system xc-) (Cheah et al., 2006), as well as lipid peroxidation mediated by the overexpression of ACSL4 (Gubern et al., 2013) and 12/15-LOX (Yigitkanli et al., 2017; Figure 1).

\section{Mechanisms of Iron Overload During Cerebral Ischemia}

Iron overload following IS is profoundly significant upon the activation of ferroptosis. Reportedly, iron overload after cerebral ischemia aggravated cell death in brain tissues, whereas blockade of iron overload elicited the opposite effect, protecting cerebral tissues from ischemic injury (Tuo et al., 2017).

Additionally, a study reported that serum levels of hepcidin and iron increased in IS patients. This indicates that hepcidin plays an important role in iron overload during cerebral ischemia (Petrova et al., 2016). The internalization and degradation of hepcidin are induced through its binding with FPN1 on the cell membrane, rendering it a negative regulator of iron release (Petrova et al., 2016). Currently, there are two potential pathways responsible for iron overload in ischemic brain tissue. The primary pathway involves increased expression of interleukin6 (IL-6) owing to ischemia. IL-6 potentiates the expression of hepcidin through the JAK/STAT3 pathway, leading to a decrease in FPN1 and, consequently, reduced iron release. This results in intracellular iron overload (Ding et al., 2011). However, the mechanism of IL-6 upregulation in cerebral ischemia remains unclear. Similarly, the upregulation of hypoxiainducible factor 1-alpha (HIF-1 $\alpha$ ) after cerebral ischemia results in iron overload, increasing transferrin receptor 1 (TFR1) expression. Considering that the TF-TFR1 pathway is potentially necessary for neurons to absorb iron, the increased iron uptake is possibly a decisive step for iron overload (Ke and Qian, 2007; Tang et al., 2008).

Moreover, inhibition of iron overload can reduce the damage caused by IS. In transient IS models, an intramuscular injection of the iron-chelating agent deferoxamine markedly reduces the volume of cerebral infarction (Xing et al., 2009). Another iron-chelating agent, 2,2-bipyridine, exerted a protective effect in a rat model of permanent cerebral ischemia (Demougeot et al., 2004). A specific iron-chelating agent, salicylaldehyde isonicotinoyl hydrazine (SIH), effectively alleviated ischemiareperfusion injury (Tuo et al., 2017). Therefore, iron overload occurs in IS and is closely related to ferroptosis, implying that iron overload is an important regulatory mechanism of ferroptosis in IS.

\section{Iron-Related Proteins and IS}

Recent studies revealed that TFR1 expression is upregulated in the brain during cerebral ischemia in rats, and Naotaifang extract reduces TFR1 expression to inhibit ferroptosis and improve brain injury (Lan et al., 2020). Paradoxically, some studies demonstrated that TFR has the opposite effect. Pretreatment with low-dose thrombin can increase the HIF- $1 \alpha$ level through the p44/42 MAPK pathway, upregulating TF and TFR expression in the brain parenchyma and reducing iron-overload-mediated cerebral ischemic injury (Hua et al., 2003). This is because the brain TF can absorb iron from the circulation, and, additionally, iron can also quickly flow from the brain into the bloodstream (Zhang and Pardridge, 2001). This may suggest that the upregulation of TFR1 leads to iron overload, and accumulation of TFR1 beyond a certain threshold will allow iron clearance and reduce iron overload (Hua et al., 2003). Nevertheless, further investigations are imperative to elucidate the comprehensive effects of TFR1. Moreover, DMT1 expression was upregulated in rats with cerebral ischemia, and DMT1 inhibitors protected against ferroptosis, reducing cerebral ischemia injury (Lan et al., 2020).

\section{AMINO ACID METABOLISM AND CEREBRAL ISCHEMIA}

\section{Expression of System xc- Is Upregulated in Cerebral Ischemia, Aggravating Injury}

Notably, glutamate excitotoxicity after cerebral ischemia has been described as ferroptosis and can be effectively inhibited by the ferroptotic inhibitor ferrostatin-1 (Dixon et al., 2012). Moreover, glutamate excitotoxicity has been proposed as a mechanism of ferroptosis (Krzyżanowska et al., 2014). Signaling through glutamate receptors located on the neuronal cytomembrane, especially the N-methyl-D-aspartate receptor (NMDAR), promotes intracellular signaling and directly induces cell death (Cheah et al., 2006). NMDAR promotes calcium influx, extracellular iron uptake (including 


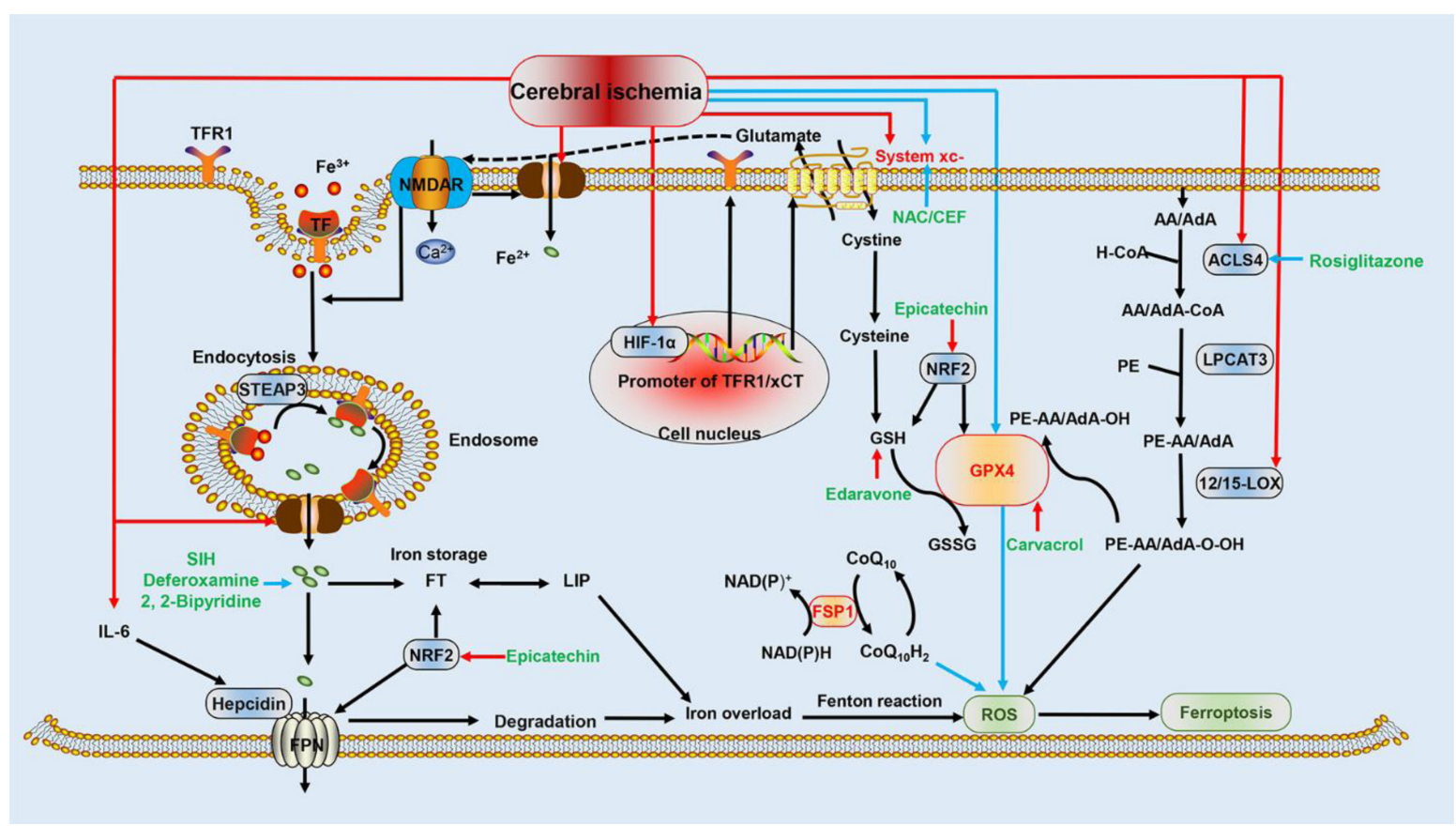

FIGURE 1 | Schematic representation of ferroptotic mechanisms following cerebral ischemia. This figure summarizes the potential mechanism of cerebral ischemia in regulating ferroptosis and the molecular mechanisms of ferroptosis: (i) the red arrows indicate that HIF-1 $\alpha$, DMT1, ACSL4, 12/15-LOX, system XC-, and IL-6 are upregulated by cerebral ischemia, which promotes ferroptosis and worsens cerebral ischemia injury; (ii) the blue arrows indicate that system XC- and GPX4 are repressed during cerebral ischemia, promoting ferroptosis and potentiating damage; (iii) the additional FSP1 pathway inhibits the formation of $\mathrm{ROS}_{\mathrm{S}}$ through $\mathrm{CoQ}_{10} \mathrm{H}_{2}$ and the Nrf2 pathway represses ferroptosis by attenuating iron overload and oxidative damage; (iv) black arrows represent the molecular mechanisms of ferroptosis; and (v) green font indicates drugs that inhibit ferroptosis and interfere with ischemic brain injury. Blue arrows indicate inhibition, and red arrows indicate stimulation. HIF-1 $\alpha$, hypoxia-inducible factor 1-alpha; DMT1, divalent metal transporter 1; ASL4, acyl-CoA synthetase long-chain family member 4; IL-6, interleukin 6; glutathione peroxidase 4; LOX, lipoxygenase; ROS, reactive oxygen species; FSP1, ferroptosis suppressor protein 1; Nrf2, nuclear factor erythroid 2-related factor 2 (Nrf2).

free iron and holotransferrin), and neuronal excitotoxicity through the NMDA-NO-Dexras1-PAP7-DMT1 pathway (Cheah et al., 2006).

System xc- is a heterodimer composed of SLC3A2 (regulatory subunit) and SLC7A11 (also known as xCT), playing a key role in the amino acid transport system (Dixon et al., 2012). In most animal models of neurological diseases, $\mathrm{xCT}$ is upregulated and leads to the release of non-synaptic glutamate, which in turn activates glutamate receptors. Excitotoxicity induced by $\mathrm{xCT}$ through glutamate receptors circumvents the antioxidant protection afforded by system xc- itself (Lewerenz et al., 2013). After the in vitro oxygen-glucose deprivation of neurons, HIF$1 \alpha$ binds to the promoter of $\mathrm{xCT}$, upregulating its expression and then inducing injury (Hsieh et al., 2017). Conversely, the downregulation of xCT expression by $\mathrm{N}$-acetylcysteine and ceftriaxone significantly reduced IS injury (Krzyżanowska et al., 2017). Neuronal excitotoxicity, also called ferroptosis, damages ischemic tissue through NMDAR activation, mediated by an increased extracellular glutamate content, mainly caused xCT after cerebral ischemia; repression of system xc- can inhibit ferroptosis to prevent damage. However, deletion of the transport subunit of system xc- does not impair the development of a young mouse brain and exerts only a marginal impact on an adult mouse brain (Sato et al., 2005), possibly induced by the presence of a substance compensating for system xc- in vivo. This rationalizes that an increased expression of system xc- in cerebral ischemia cannot inhibit but can promote the occurrence of ferroptosis. Intriguingly, a study has demonstrated that increased expression of the $p 53$ gene in rats repressed $\mathrm{xCT}$ and resulted in ferroptosis during cerebral ischemia (Lan et al., 2020). These seemingly contradictory results suggest that there exists an intricate relationship between $\mathrm{xCT}$, cerebral ischemia, and ferroptosis. Therefore, additional research in this direction is needed in the future.

\section{Upregulation of GPX4 and GSH Can Improve Cerebral Ischemic Injury}

GPX4 and GSH are endogenous inhibitors of ferroptosis, and their content is closely related to cerebral ischemia. Presently, it is believed that the loss of GPX4 function is crucial for ferroptosis owing to its pivotal role in the inhibition of lipid peroxidation (Stockwell et al., 2017). Furthermore, the inhibition of ferroptosis by GPX4 suppression reduced brain damage in rats subjected to ischemic damage. In ischemic rat models, significantly low levels of GPX4 resulted in ferroptosis, whereas high levels of GPX4 could improve brain injury (Lan et al., 2020). Selenium promotes the expression of GPX4 by activating the transcription factors TFAP2c and Sp1, effectively inhibiting ferroptosis, and alleviating IS injury (Alim et al., 2019). Carvacrol, a drug indicated for 
the treatment of cerebral ischemia, is beneficial in inhibiting ferroptosis in gerbils by potentiating the expression of GPX4 and attenuating hippocampal neuronal damage during cerebral ischemia-reperfusion injury (Guan et al., 2019).

GSH is essential for GPX4 activity; furthermore, it is the only intracellular ligand of $\mathrm{Fe}^{2+}$ that prevents the generation of highly toxic hydroxyl radicals, thereby inhibiting ferroptosis (Philpott and Ryu, 2014). Moreover, promoting GSH generation is beneficial for ameliorating IS. As a clinically approved drug for acute IS, edaravone has been proposed to combat ferroptosis, especially in cystine deficiency (Homma et al., 2019). In summary, enhanced GPX4 expression and GSH synthesis can suppress ferroptosis and mitigate ischemic brain damage. However, currently, researchers are focusing on the effect of GPX4 and GSH rather than on the upstream mechanism of cerebral ischemia.

\section{LIPID METABOLISM AND CEREBRAL ISCHEMIA}

\section{Inhibition of ACSL4 Inhibits Ferroptosis and Improves Cerebral Ischemic Injury}

ACSL4 and LPCAT3 are involved in the remodeling of phosphatidylethanolamine, which affects the synthesis of lipid peroxides. However, only ACSL4 is upregulated and participates in ischemic injury. Reportedly, the inhibition of ACSL4 can prevent ferroptosis; thus, cell death and intestinal injury are alleviated (Li et al., 2019). Additionally, ACSL4 is widely expressed in brain tissues and is increased during cerebral ischemia, with an increased expression of miRNA-347 upregulating ACSL4 at the post-transcriptional level, and mediating neuronal death (Gubern et al., 2013). Rosiglitazone can selectively repress the activity of ACSL4, thereby preventing ferroptosis in the neurons of cerebral ischemic models and protecting brain function (Sayan-Ozacmak et al., 2012). In summary, the upregulation of ACSL4 after cerebral ischemia induces ferroptosis in neurons, leading to IS injury.

\section{Inhibition of Lox Can Inhibit Ferroptosis and Improve Cerebral Ischemic Injury}

LOX is the key enzyme catalyzing PUFAs to initiate lipid peroxidation and ferroptosis (Lei et al., 2019), highly expressed after cerebral ischemia, and LOX inhibitors can suppress ferroptosis (Lei et al., 2019) to attenuate injury (Karatas et al., 2018). One LOX inhibitor, called ML351, exerted a protective effect in cerebral ischemia-reperfusion injury (Tuo et al., 2017). Furthermore, there are several subtypes of LOX, including 12/15LOX. In animal models of brain ischemia, 12/15-LOX expression increases, and the inhibition of $12 / 15$-LOX can reduce the rate of the neuronal death, improving recovery (Karatas et al., 2018). Previous studies have shown that suppressing 12/15-LOX can result in the inhibition of ferroptosis (Li et al., 2018). To date, it remains unclear whether multiple subtypes work in conjunction or whether only one subtype is sufficient to initiate lipid peroxidation and ferroptosis, necessitating further study.
In conclusion, the mechanism of ferroptosis during cerebral ischemia is related to the accumulation of intracellular $\mathrm{Fe}^{2+}$, lipid peroxides, and oxidative damage induced by the downregulation of GPX4. The specific factors are as follows: the increased expression of HIF-1 $\alpha$ enhances the expression of TFR1 and $\mathrm{xCT}$, both of which potentiate iron uptake and iron overload; increased IL-6 induces an increase in hepcidin content, which suppresses iron excretion and leads to iron overload; an unknown mechanism triggers the downregulation of GPX4; and increased expression of ACSL4 and 12/15-LOX leads to lipid peroxidation in the cell membrane.

\section{OTHER MECHANISMS: INHIBITION OF FERROPTOSIS BY Nrf2 IS EXPECTED TO IMPROVE IS INJURY}

Nuclear factor erythroid-2-related factor $2(\mathrm{Nrf} 2)$ is an important regulator of the cellular antioxidant defense system. A study demonstrated that the appropriate activation of Nrf2 promotes the alleviation of cerebral ischemic injury (Ma, 2013). Several genes targeted by Nrf2 are strongly associated with the progression of ferroptosis, including ferritin heavy chain 1 , FPN1, GSH, and GPX4 (Ma, 2013; Kerins and Ooi, 2018). Furthermore, it has been clarified that the expression level of Nrf2 is dependent on ferroptosis sensitivity. The enhanced expression of Nrf2 can inhibit ferroptosis. Conversely, the inhibition of Nrf2 expression induces ferroptosis (Sun et al., 2016). Nrf2 inhibits ferroptosis by promoting the expression of GSH and GPX4, thus enhancing antioxidant function. Moreover, Nrf2 promotes the expression of ferritin and FPN1 to store and secrete free iron concurrently, thus reducing the accumulation of iron in cells and preventing ferroptosis (Yang et al., 2017). Therefore, Nrf2 is a promising antiferroptosis target.

Currently, evidence clarifying the mechanism by which Nrf2 directly affects ferroptosis during stroke is scarce. However, there are several indications regarding the role of the Nrf2 pathway in IS. Reportedly, epicatechin can cross the blood-brain barrier to exert a protective effect in transient IS through the Nrf2 pathway (Shah et al., 2010). However, related laboratory and clinical data elucidating this mechanism remain lacking.

\section{CONCLUDING REMARKS AND PERSPECTIVES}

Cerebral ischemia can induce ferroptosis and, in turn, ferroptosis aggravates cerebral ischemic injury; inhibiting ferroptosis can alleviate this injury. However, the regulatory mechanisms involved with ferroptosis in cerebral ischemia, including iron overload and regulatory mechanisms upstream of GPX4, 12/15LOX, and Nrf2, remain unclear; in particular, whether ferroptosis causes cell death directly or by other means requires further investigation. Additionally, the relationship between ferroptosis and other cell death mechanisms, such as apoptosis and pyroptosis, in cerebral ischemia, is an important direction 
for future research. The resolution of these obstacles will provide crucial support for the development of ferroptosis as a target in cerebral ischemia interventions and an important direction for the study of pathological mechanisms involved in cerebral ischemia.

\section{AUTHOR CONTRIBUTIONS}

$\mathrm{XS}$ and BT reviewed the literature and drafted the manuscript. $\mathrm{BL}, \mathrm{HT}$, and BT finalized the manuscript and provided suggestions for improvements. All authors participated in designing the concept of this manuscript.

\section{REFERENCES}

Alim, I., Caulfield, J. T., Chen, Y., Swarup, V., Geschwind, D. H., Ivanova, E., et al. (2019). Selenium drives a transcriptional adaptive program to block ferroptosis and treat stroke. Cell 177, 1262-1279. doi: 10.1016/j.cell.2019.03.032

Aoyama, K., and Nakaki, T. (2015). Glutathione in cellular redox homeostasis: association with the excitatory amino acid carrier 1 (EAAC1). Molecules 20, 8742-8758. doi: 10.3390/molecules20058742

Bersuker, K., Hendricks, J. M., Li, Z., Magtanong, L., Ford, B., Tang, P. H., et al. (2019). The CoQ oxidoreductase FSP1 acts parallel to GPX4 to inhibit ferroptosis. Nature 575, 688-692. doi: 10.1038/s41586-019-1705-2

Bridges, R., Lutgen, V., Lobner, D., and Baker, D. A. (2012). Thinking outside the cleft to understand synaptic activity: contribution of the cystine-glutamate antiporter (System $\mathrm{Xc}_{\mathrm{c}}$ ) to normal and pathological glutamatergic signaling. Pharmacol. Rev. 64, 780-802. doi: 10.1124/pr.110.003889

Chan, P. H. (2001). Reactive oxygen radicals in signaling and damage in the ischemic brain. J. Cereb. Blood Flow Metab. 21, 2-14. doi: 10.1097/00004647200101000-00002

Cheah, J. H., Kim, S. F., Hester, L. D., Clancy, K. W., Patterson, S. E., Papadopoulos, V., et al. (2006). NMDA receptor-nitric oxide transmission mediates neuronal iron homeostasis via the GTPase Dexras1. Neuron 51, 431-440. doi: 10.1016/j. neuron.2006.07.011

Chen, L., Hambright, W. S., Na, R., and Ran, Q. (2015). Ablation of the ferroptosis inhibitor glutathione peroxidase 4 in neurons results in rapid motor neuron degeneration and paralysis. J. Biol. Chem. 290, 28097-28106. doi: 10.1074/jbc. M115.680090

Chuanhong, W., Wenwen, Z., Jie, Y., Shaojing, L., Ligen, L., and Xiuping, C. (2018). Induction of ferroptosis and mitochondrial dysfunction by oxidative stress in PC12 Cells. Sci. Rep. 8:574. doi: 10.1038/s41598-017-18935-1

Conrad, M., Angeli, J. P., Vandenabeele, P., and Stockwell, B. R. (2016). Regulated necrosis: disease relevance and therapeutic opportunities. Nat Rev. Drug Discov. 15, 348-366. doi: 10.1038/nrd.2015.6

DeGregorio-Rocasolano, N., Martí-Sistac, O., and Gasull, T. (2019). Deciphering the iron side of stroke: neurodegeneration at the crossroads between iron dyshomeostasis, excitotoxicity, and ferroptosis. Front. Neurosci. 13:85. doi: 10. 3389/fnins.2019.00085

Demougeot, C., Van Hoecke, M., Bertrand, N., Prigent-Tessier, A., Mossiat, C., Beley, A., et al. (2004). Cytoprotective efficacy and mechanisms of the liposoluble iron chelator 2,2'-dipyridyl in the rat photothrombotic ischemic stroke model. J. Pharmacol. Exp. Ther. 311, 1080-1087. doi: 10.1124/jpet.104. 072744

Ding, H., Yan, C. Z., Shi, H., Zhao, Y. S., Chang, S. Y., Yu, P., et al. (2011). Hepcidin is involved in iron regulation in the ischemic brain. PLoS One 6:e25324. doi: 10.1371/journal.pone.0025324

Dixon, S. J., Lemberg, K. M., Lamprecht, M. R., Skouta, R., Zaitsev, E. M., Gleason, C. E., et al. (2012). Ferroptosis: an iron-dependent form of nonapoptotic cell death. Cell 149, 1060-1072. doi: 10.1016/j.cell.2012.03.042

Dixon, S. J., Winter, G. E., Musavi, L. S., Lee, E. D., Snijder, B., Rebsamen, M., et al. (2015). Human haploid cell genetics reveals roles for lipid metabolism

\section{FUNDING}

This work was supported by the National Natural Science Foundation of China (Nos. 81503385 and 81874508), the Scientific Research Fund of Hunan Provincial Education Department (No. 19B436), the Hunan Provincial Innovation Foundation for Postgraduate (No. CX2018B515), the Open Fund of the Domestic First-Class Discipline Construction Project of Integrated Traditional Chinese and Western Medicine of Hunan University of Chinese Medicine (No. 2018ZXYJH35), and the First-Class Discipline Construction Project of Basic Medicine in the 13th Five-Year Plan of Hunan University of Chinese Medicine (06).

genes in nonapoptotic cell death. ACS Chem Biol. 10, 1604-1609. doi: 10.1021/ acschembio. 5 b00245

Do Van, B., Gouel, F., Jonneaux, A., Timmerman, K., Gelé, P., Pétrault, M., et al. (2016). Ferroptosis, a newly characterized form of cell death in Parkinson's disease that is regulated by PKC. Neurobiol. Dis 94, 169-178. doi: 10.1016/j.nbd. 2016.05.011

Doll, S., Freitas, F. P., Shah, R., Aldrovandi, M., da Silva, M. C., Ingold, I., et al. (2019). FSP1 is a glutathione-independent ferroptosis suppressor. Nature 575, 693-698. doi: 10.1038/s41586-019-1707-0

Doll, S., Proneth, B., Tyurina, Y. Y., Panzilius, E., Kobayashi, S., Ingold, I., et al. (2017). ACSL4 dictates ferroptosis sensitivity by shaping cellular lipid composition. Nat. Chem. Biol. 13, 91-98. doi: 10.1038/nchembio.2239

Dolma, S., Lessnick, S. L., Hahn, W. C., and Stockwell, B. R. (2003). Identification of genotype-selective antitumor agents using synthetic lethal chemical screening in engineered human tumor cells. Cancer Cell 3, 285-296. doi: 10.1016/s15356108(03)00050-3

Donovan, A., Brownlie, A., Zhou, Y., Shepard, J., Pratt, S. J., and Moynihan, J. (2000). Positional cloning of zebrafish ferroportin1 identifies a conserved vertebrate iron exporter. Nature 403, 776-781. doi: 10.1038/35001596

Eran, A., Jérôme, S., Patricia, B., and Brent, R. S. (2018). Modeling the effects of lipid peroxidation during ferroptosis on membrane properties. Sci. Rep. 8:5155. doi: 10.1038/s41598-018-23408-0

Gaschler, M. M., and Stockwell, B. R. (2016). Lipid peroxidation in cell death. Biochem. Biophys. Res. Commun. 482, 419-425.

Gascón, S., Murenu, E., Masserdotti, G., Ortega, F., Russo, G. L., Petrik, D., et al. (2016). Identification and successful negotiation of a metabolic checkpoint in direct neuronal reprogramming. Cell Stem Cell 18, 396-409. doi: 10.1016/j.stem. 2015.12.003

Guan, X., Li, X., Yang, X., Yan, J., Shi, P., Ba, L., et al. (2019). The neuroprotective effects of carvacrol on ischemia/ reperfusion-induced hippocampal neuronal impairment by ferroptosis mitigation. Life Sci. 235:116795. doi: 10.1016/j.lfs. 2019.116795

Gubern, C., Camós, S., Ballesteros, I., Rodríguez, R., Romera, V. G., Cañadas, R., et al. (2013). miRNA expression is modulated over time after focal ischaemia: up-regulation of miR347 promotes neuronal apoptosis. FEBS J. 280, 6233-6246. doi: $10.1111 /$ febs.12546

Guiney, S. J., Adlard, P. A., Bush, A. I., Finkelstein, D. I., and Ayton, S. (2017). Ferroptosis and cell death mechanisms in Parkinson's disease. Neurochem. Int. 104, 34-48. doi: 10.1016/j.neuint.2017.01.004

Hambright, W. S., Fonseca, R. S., Chen, L., Na, R., and Ran, Q. (2017). Ablation of ferroptosis regulator glutathione peroxidase 4 in forebrain neurons promotes cognitive impairment and neurodegeneration. Redox. Biol. 12, 8-17. doi: 10. 1016/j.redox.2017.01.021

Hentze, M. W., Muckenthaler, M. U., Galy, B., and Camaschella, C. (2010). Two to tango: regulation of mammalian iron metabolism. Cell 142, 24-38. doi: 10.1016/j.cell.2010.06.028

Homma, T., Kobayashi, S., Sato, H., and Fujii, J. (2019). Edaravone, a free radical scavenger, protects against ferroptotic cell death in vitro. Exp. Cell Res. 384:11592. doi: 10.1016/j.yexcr.2019.111592 
Hsieh, C. H., Lin, Y. J., Chen, W. L., Huang, Y. C., Chang, C. W., Cheng, F. C., et al. (2017). HIF-1 $\alpha$ triggers long-lasting glutamate excitotoxicity via system Xc- in cerebral ischaemia-reperfusion. J. Pathol. 241, 337-349. doi: 10.1002/path.4838

Hua, Y., Keep, R. F., Hoff, J. T., and Xi, G. (2003). Thrombin preconditioning attenuates brain edema induced by erythrocytes and iron. J. Cereb. Blood Flow Metab. 23, 1448-1454. doi: 10.1097/01.WCB.0000090621.86921.D5

Imai, H., Graham, D. I., Masayasu, H., and Macrae, I. M. (2003). Antioxidant ebselen reduces oxidative damage in focal cerebral ischemia. Free Radic. Biol. Med. 34, 56-63. doi: 10.1016/s0891-5849(02)01180-2

Jose, P. F. A., Manuela, S., Bettina, P., Yulia, Y. T., Vladimir, A. T., and Victoria, J. H. (2014). Inactivation of the ferroptosis regulator Gpx4 triggers acute renal failure in mice. Nat. Cell Biol. 16, 1180-1191. doi: 10.1038/ncb3064

Kagan, V. E., Mao, G., Qu, F., Angeli, J. P., Doll, S., Croix, C. S., et al. (2017). Oxidized arachidonic and adrenic PEs navigate cells to ferroptosis. Nat. Chem. Biol. 13, 81-90. doi: 10.1038/nchembio.2238

Karatas, H., Eun Jung, J., Lo, E. H., and van Leyen, K. (2018). Inhibiting 12/15lipoxygenase to treat acute stroke in permanent and tPA induced thrombolysis models. Brain Res. 1678, 123-128. doi: 10.1016/j.brainres.2017.10.024

Ke, Y., and Qian, Z. M. (2007). Brain iron metabolism: neurobiology and neurochemistry. Prog. Neurobiol. 83, 149-173. doi: 10.1016/j.pneurobio.2007. 07.009

Kerins, M. J., and Ooi, A. (2018). The roles of NRF2 in modulating cellular iron homeostasis. Antioxid. Redox Signal. 29, 1756-1773. doi: 10.1089/ars.2017.7176

Krzyżanowska, W., Pomierny, B., Bystrowska, B., Pomierny-Chamioło, L., Filip, M., Budziszewska, B., et al. (2017). Ceftriaxone- and N-acetylcysteine-induced brain tolerance to ischemia: influence on glutamate levels in focal cerebral ischemia. PLoS One 12:e0186243. doi: 10.1371/journal.pone.0186243

Krzyżanowska, W., Pomierny, B., Filip, M., and Joanna, P. (2014). Glutamate transporters in brain ischemia: to modulate or not? Acta Pharmacol. Sin. 35, 444-462. doi: 10.1038/aps.2014.1

Lan, B., Ge, J. W., Cheng, S. W., Zheng, X. L., Liao, J., He, C., et al. (2020). Extract of Naotaifang, a compound Chinese herbal medicine, protects neuron ferroptosis induced by acute cerebral ischemia in rats. J Integr. Med. 55(Suppl. 1):1803. doi: $10.1016 /$ j.joim.2020.01.008

Le, J., Ning, K., Tongyuan, L., ShangJui, W., Tao, S., Hanina, H., et al. (2015). Ferroptosis as a p53-mediated activity during tumour suppression. Nature 520, 57-62. doi: 10.1038/nature14344

Lee, J. M., Zipfel, G. J., and Choi, D. W. (1999). The changing landscape of ischaemic brain injury mechanisms. Nature 399, A7-A14. doi: 10.1038/399a007

Lei, P., Bai, T., and Sun, Y. (2019). Mechanisms of ferroptosis and relations with regulated cell death: a review. Front. Physiol. 10:139. doi: 10.3389/fphys.2019. 00139

Leslie, M., and Scott, J. D. (2018). Ferroptosis and brain injury. Dev. Neurosci. 40, 382-395. doi: 10.1159/000496922

Lewerenz, J., Hewett, S. J., Huang, Y., Lambros, M., Gout, P. W., Kalivas, P. W., et al. (2013). The cystine/glutamate antiporter system X(c) (-) in health and disease: from molecular mechanisms to novel therapeutic opportunities. Antioxid. Redox Signal. 18, 522-555. doi: 10.1089/ars.2011.4391

Li, Q., Han, X., Lan, X., Gao, Y., Wan, J., Durham, F., et al. (2017). Inhibition of neuronal ferroptosis protects hemorrhagic brain. JCI Insight 2:e90777. doi: 10.1172/jci.insight.90777

Li, Q. Q., Li, Q., Jia, J. N., Liu, Z. Q., Zhou, H. H., and Mao, X. Y. (2018). 12/15 lipoxygenase: a crucial enzyme in diverse types of cell death. Neurochem. Int. 118, 34-41. doi: 10.1016/j.neuint.2018.04.002

Li, Y., Feng, D., Wang, Z., Zhao, Y., Sun, R., Tian, D., et al. (2019). Ischemiainduced ACSL4 activation contributes to ferroptosis-mediated tissue injury in intestinal ischemia/reperfusion. Cell Death Differ. 26, 2284-2299. doi: 10.1038/ s41418-019-0299-4

Linkermann, A., Skouta, R., Himmerkus, N., Mulay, S. R., Dewitz, C., De Zen, F., et al. (2014a). Synchronized renal tubular cell death involves ferroptosis. Proc. Natl. Acad. Sci. U.S.A. 111, 16836-16841. doi: 10.1073/pnas.1415518111

Linkermann, A., Stockwell, B. R., Krautwald, S., and Anders, H. J. (2014b). Regulated cell death and inflammation: an auto-amplification loop causes organ failure. Nat. Rev. Immunol. 14, 759-767. doi: 10.1038/nri3743

Ma, Q. (2013). Role of nrf2 in oxidative stress and toxicity. Annu. Rev. Pharmacol. Toxicol. 53, 401-426. doi: 10.1146/annurev-pharmtox-011112-140320

Michael, M. G., and Brent, R. S. (2017). Lipid peroxidation in cell death. Biochem. Biophys. Res. Commun. 482, 419-425.
Neitemeier, S., Jelinek, A., Laino, V., Hoffmann, L., Eisenbach, I., Eying, R., et al. (2017). BID links ferroptosis to mitochondrial cell death pathways. Redox Biol. 12, 558-570. doi: 10.1016/j.redox.2017.03.007

Newton, K., Dugger, D. L., Maltzman, A., Greve, J. M., Hedehus, M., Martin-McNulty, B., et al. (2016). RIPK3 deficiency or catalytically inactive RIPK1 provides greater benefit than MLKL deficiency in mouse models of inflammation and tissue injury. Cell Death Differ. 23, 1565-1576. doi: 10.1038/ cdd. 2016.46

Petrova, J., Manolov, V., Vasilev, V., Tzatchev, K., and Marinov, B. (2016). Ischemic stroke, inflammation, iron overload - Connection to a hepcidin. Int. J. Stroke 11, N16-N17. doi: 10.1177/1747493015607509

Philpott, C. C., and Ryu, M. S. (2014). Special delivery: distributing iron in the cytosol of mammalian cells. Front. Pharmacol. 5:173. doi: 10.3389/fphar.2014. 00173

Richardson, D. R., and Ponka, P. (1997). The molecular mechanisms of the metabolism and transport of iron in normal and neoplastic cells. Biochim. Biophys. Acta 1331, 1-40. doi: 10.1016/s0304-4157(96)00014-7

Sato, H., Shiiya, A., Kimata, M., Maebara, K., Tamba, M., Sakakura, Y., et al. (2005). Redox imbalance in cystine/glutamate transporter-deficient mice. J. Biol. Chem. 280, 37423-37429. doi: 10.1074/jbc.M506439200

Sayan-Ozacmak, H., Ozacmak, V. H., Barut, F., and Jakubowska-Dogru, E. (2012). Rosiglitazone treatment reduces hippocampal neuronal damage possibly through alleviating oxidative stress in chronic cerebral hypoperfusion. Neurochem. Int. 61, 287-290. doi: 10.1016/j.neuint.2012.05.011

Shah, Z. A., Li, R. C., Ahmad, A. S., Kensler, T. W., Yamamoto, M., Biswal, S., et al. (2010). The flavanol (-)-epicatechin prevents stroke damage through the Nrf2/HO1 pathway. J. Cereb. Blood Flow Metab. 30, 1951-1961. doi: 10.1038/ jcbfm. 2010.53

Shimada, K., Skouta, R., Kaplan, A., Yang, W. S., Hayano, M., Dixon, S. J., et al. (2016). Global survey of cell death mechanisms reveals metabolic regulation of ferroptosis. Nat. Chem. Biol. 12, 497-503. doi: 10.1038/nchembio.2079

Shintoku, R., Takigawa, Y., Yamada, K., Kubota, C., Yoshimoto, Y., Takeuchi, T., et al. (2017). Lipoxygenase-mediated generation of lipid peroxides enhances ferroptosis induced by erastin and RSL3. Cancer Sci. 108, 2187-2194. doi: $10.1111 /$ cas. 13380

Skouta, R., Dixon, S. J., Wang, J., Dunn, D. E., Orman, M., Shimada, K., et al. (2014). Ferrostatins inhibit oxidative lipid damage and cell death in diverse disease models. J. Am. Chem. Soc. 136, 4551-4556. doi: 10.1021/ja411006a

Stockwell, B. R., Friedmann Angeli, J. P., Bayir, H., Bush, A. I., Conrad, M., Dixon, S. J., et al. (2017). Ferroptosis: a regulated cell death nexus linking metabolism, redox biology, and disease. Cell 171, 273-285. doi: 10.1016/j.cell.2017. 09.021

Sun, X., Ou, Z., Chen, R., Niu, X., Chen, D., Kang, R., et al. (2016). Activation of the p62-Keap1-NRF2 pathway protects against ferroptosis in hepatocellular carcinoma cells. Hepatology 63, 173-184. doi: 10.1002/hep.28251

Sun, Y., Chen, P., Zhai, B., Zhang, M., Xiang, Y., Fang, J., et al. (2020). The emerging role of ferroptosis in inflammation. Biomed. Pharmacother. 127:110108. doi: 10.1016/j.biopha.2020.110108

Tang, W. H., Wu, S., Wong, T. M., Chung, S. K., and Chung, S. S. (2008). Polyol pathway mediates iron-induced oxidative injury in ischemic-reperfused rat heart. Free Radic. Biol. Med. 45, 602-610. doi: 10.1016/j.freeradbiomed.2008. 05.003

Toyokuni, S., Ito, F., Yamashita, K., Okazaki, Y., and Akatsuka, S. (2017). Iron and thiol redox signaling in cancer: an exquisite balance to escape ferroptosis. Free Radic. Biol. Med. 108, 610-626. doi: 10.1016/j.freeradbiomed.2017.04.024

Tuo, Q. Z., Lei, P., Jackman, K. A., Li, X. L., Xiong, H., Li, X. L., et al. (2017). Tau-mediated iron export prevents ferroptotic damage after ischemic stroke. Mol. Psychiatry 22, 1520-1530. doi: 10.1038/mp. 2017.171

Xie, Y., Hou, W., Song, X., Yu, Y., Huang, J., Sun, X., et al. (2016). Ferroptosis: process and function. Cell Death Differ. 23, 369-379. doi: 10.1038/cdd.2015.158

Xing, Y., Hua, Y., Keep, R. F., and Xi, G. (2009). Effects of deferoxamine on brain injury after transient focal cerebral ischemia in rats with hyperglycemia. Brain Res. 1291, 113-121. doi: 10.1016/j.brainres.2009.07.032

Yan, N., and Zhang, J. J. (2019). The emerging roles of ferroptosis in vascular cognitive impairment. Front. Neurosci. 13:811. doi: 10.3389/fnins.2019.00811

Yang, W. S., Kim, K. J., Gaschler, M. M., Patel, M., Shchepinov, M. S., and Stockwell, B. R. (2016). Peroxidation of polyunsaturated fatty acids by lipoxygenases drives 
ferroptosis. Proc Natl. Acad. Sci. U.S.A. 113, E4966-E4975. doi: 10.1073/pnas. 1603244113

Yang, W. S., SriRamaratnam, R., Welsch, M. E., Shimada, K., Skouta, R., Viswanathan, V. S., et al. (2014). Regulation of ferroptotic cancer cell death by GPX4. Cell 156, 317-331. doi: 10.1016/j.cell.2013.12.010

Yang, X., Park, S. H., Chang, H. C., Shapiro, J. S., Vassilopoulos, A., Sawicki, K. T., et al. (2017). Sirtuin 2 regulates cellular iron homeostasis via deacetylation of transcription factor NRF2. J. Clin. Invest. 127, 1505-1516. doi: 10.1172/ JCI88574

Yigitkanli, K., Zheng, Y., Pekcec, A., Lo, E. H., and van Leyen, K. (2017). Increased 12/15-lipoxygenase leads to widespread brain injury following global cerebral ischemia. Transl. Stroke Res. 8, 194-202. doi: 10.1007/s12975-0160509-Z

Zhang, Y., and Pardridge, W. M. (2001). Rapid transferrin efflux from brain to blood across the blood-brain barrier. J. Neurochem. 76, 1597-1600. doi: 10. 1046/j.1471-4159.2001.00222.x
Zille, M., Karuppagounder, S. S., Chen, Y., Gough, P. J., Bertin, J., Finger, J., et al. (2017). Neuronal death after hemorrhagic stroke in vitro and in vivo shares features of ferroptosis and necroptosis. Stroke 48, 1033-1043. doi: 10.1161/ STROKEAHA.116.015609

Conflict of Interest: The authors declare that the research was conducted in the absence of any commercial or financial relationships that could be construed as a potential conflict of interest.

Copyright (c) 2020 She, Lan, Tian and Tang. This is an open-access article distributed under the terms of the Creative Commons Attribution License (CC BY).

The use, distribution or reproduction in other forums is permitted, provided the original author(s) and the copyright owner(s) are credited and that the original publication in this journal is cited, in accordance with accepted academic practice. No use, distribution or reproduction is permitted which does not comply with these terms. 